\title{
analyse de la sécurité des fondations superficielles vis-à-vis d'un défaut de portance : effet de la variabilité spatiale des paramètres du sol
}

\author{
safety analysis of shallow foundations \\ towards the bearing capacity: \\ effect of the soil parameters spatial variability
}

\author{
Jean-Louis FAVRE \\ Professeur, Laboratoire de Mécanique des Sols-Structures, C.N.R.S. UA $850^{*}$ \\ Bernard GENEVOIS \\ Assistant-Professeur, Département de Génie Civil * *
}

\section{Résumé}

La prise en compte de la variabilité spatiale des paramètres du sol conduit à des dispersions de la charge ultime dix à cent fois plus faibles que celles trouvées par le calcul classique de fiabilité appliqué à l'équation de portance. Les probabilités de ruine calculées se trouvent ainsi ramenées dans un domaine réaliste. On utilise un code de calcul par Éléments Finis avec une loi élastoplastique et une procédure simplifiée de simulation. On mène une étude de sensibilité en fonction des moyennes, variances, autocorrélations et corrélations de deux paramètres: l'angle de frottement interne et le module d'élasticité.

\section{Abstract}

The coefficient of variation of the bearing capacity is very large using the classical reliability theory applied to the bearing capacity equation. Taking into account the spatial variability of soil properties we find a coefficient of variation ten to one hundred times smaller. The probabilities of failure are thus reduced to realistic values. We have used a Finite Elements code with an elastoplastic law and a simplified simulation procedure. A sensitivity study is run in regard to the means, variances, autocorrelations and correlations of two parameters: the internal friction angle and the elastic modulus.

\footnotetext{
- École Centrale de Paris, 92295 Chatenay-Malabry Cedex, France.
}

* Université de Permanbuco, 50000 Recife, PE, Brésil. 


\section{INTRODUCTION}

L'analyse de la sécurité en géotechnique se mène traditionnellement de façon déterministe à l'aide d'un paramètre unique, le facteur de sécurité. Celui-ci prend en compte un grand nombre d'incertitudes et d'aléas sur les actions, les propriétés mécaniques, les modèles de loi de comportement et calcul, l'exécution, l'évolution de la structure avec le temps, etc., sans pouvoir les séparer et les quantifier. MEYERHOF (1977) a proposé des coefficients de sécurité partiels pour pallier à cet inconvénient, mais leur usage est peu répandu.

Les méthodes probabilistes ont connu un développement faible malgré les travaux de recherche menés depuis les années 70 , travaux dont rendent compte, pour l'essentiel, les différentes conférences internationales sur les "Applications des Statistiques et des Probabilités à l'Ingénierie des Sols et des Structures » (1.C.A.S.P., 1971, 1975, 1979, 1983). D'autre part, MAGNAN et BAGHERY (1982) ont publié une synthèse des différentes avancées obtenues grâce à ces méthodes.

Celles-ci se fondent sur la théorie de la fiabilité développée dans le domaine des structures et dont on rappelle le principe très succinctement ci-après afin de dégager les concepts essentiels à l'éclairage de nos résultats.

\section{PRINCIPE DE LA THÉORIE DE LA FIABILITÉ POUR LES STRUCTURES}

Soit $\mathrm{S}$, la sollicitation agissante transmise à un élément d'une structure soumise aux actions A.

Soit $\mathrm{R}$, la sollicitation résistante de cet élément de structure.

$\mathrm{S}$ et $\mathrm{R}$ sont des variables aléatoires représentant les moments et les efforts dans une section de l'élément. La probabilité de ruine de l'élément est alors définie par:

$$
\mathrm{p}_{t}=\operatorname{Prob}\{\mathrm{R}-\mathrm{S} \leqslant 0\}
$$

ou encore, si les variables $\mathrm{R}$ et $\mathrm{S}$ sont indépendantes et de lois de distribution et de répartition $f_{R}, f_{S}, F_{R}, F_{S}$ connues:

$$
p_{\mathrm{t}}=\int_{-\infty}^{+\infty} F_{R}(x) f_{S}(x) d x .
$$

De façon plus générale, soient $X_{1}$ les variables aléatoires ou variables de base entrant dans le calcul de $R$ et $S$ de loi de distribution conjointe $\mathrm{f}_{\mathrm{X}}\left(\mathrm{x}_{1}, \ldots \mathrm{x}_{n}\right)$ et $\mathrm{g}(\mathrm{X})=$ $\mathrm{R}-\mathrm{S}$ la fonction de performance, avec $\mathrm{X}$ le vecteur des variables $\mathrm{X}_{i}$. Alors:

$$
\begin{gathered}
p_{f}=\int_{\bar{D}} f_{X}\left(x_{1}, \ldots x_{n}\right) d x_{1} \ldots d x_{n} \\
\bar{D}=\left\{x_{1} \mid g\left(x_{1}\right) \leqslant 0\right\}
\end{gathered}
$$

$\overline{\mathrm{D}}$ : domaine de ruine dans l'espace des variables $\mathrm{x}_{i}$. HASOFER et LIND (1974) ont défini l'indice de sécurité $\beta$, invariant vis-à-vis de la forme de l'équation de performance, comme la plus courte distance de l'origine à l'équation de performance $\mathrm{g}(\mathrm{X})=0$ dans l'espace des variables transformées (elles sont alors gaussiennes et indépendantes). $\beta$ est une mesure exacte de la probabilité de ruine si la transformée de l'équation de performance est linéaire :

$$
p_{i}=\Phi(-\beta)
$$

$\Phi$ : fonction de répartition de la variable gaussienne d'espérance nulle et de variance 1 .

BENJAMIN et CORNELL (1970) avaient précédemment défini un indice approché de sécurité :

$$
\beta=\frac{E[g(X)]}{\sigma g(x)}
$$

$E[g(X)]=\int_{\mathbb{R}^{n}} g(X) f_{X} d X$; espérance mathématique de la fonction $\mathrm{g}(\mathrm{X})$.

$\left.\sigma_{g}^{2}(X)=\operatorname{VAR}[g(X)]=\int_{\mathbb{R}^{n}} \lg (X)-E[g(X)]\right\}^{2} f_{X} d X$ variance de la fonction $\mathrm{g}(\mathrm{X})$.

Cet indice correspond exactement à celui de HASOFER-LIND si les variables $\mathrm{X}_{i}$ sont gaussiennes et indépendantes.

\section{UTILISATION DE L'INDICE DE SÉCURITÉ DANS LE CAS DES FONDATIONS SUPERFICIELLES}

SCHULTZE (1977) a éclairé le problème de la sécurité à la portance des fondations superficielles grâce à une utilisation simplifiée de lindice $\beta$ qui permet de relier directement ce dernier au coefficient de sécurité $\mathrm{F}$.

Soit $\mathrm{q}_{1}$, la résistance au mètre linéaire sous une semelle filante de largeur $\mathrm{B}$.

$$
q_{L}=\frac{1}{2} \gamma B^{2} N_{\gamma}+B q_{0} N_{q}+B C N_{C}
$$

$\gamma$ : poids volumique du sol.

$\mathrm{q}_{\mathrm{o}}$ : charge additive au mètre linéaire de part et d'autre de la fondation.

$\mathrm{N}_{\gamma}, \mathrm{N}_{\mathrm{q}}, \mathrm{N}_{\mathrm{c}}$ : facteurs de portance dépendant de l'angle de frottement interne $\varnothing$ du sol.

C : cohésion du sol.

SCHULTZE se place dans le cas où la sollicitation appliquée q, due seulement au poids propre et aux charges permanentes, est de dispersion négligeable devant la dispersion de la résistance ultime. En effet, la dispersion de la cohésion est souvent très importante $\left(\mathrm{CV}_{\mathrm{C}}\right.$ de 30 à $\left.40 \%{ }^{1}\right)$; d'autre part celle de l'angle de frottement, plus faible (de l'ordre de 15 à $20 \%$ ), introduit, par le calcul, une forte dispersion des facteurs de portance (de l'ordre de 30 à $40 \%$ ). Il est donc communément admis que la dispersion de la résistance ultime est de l'ordre de 40 à $50 \%$.

L'hypothèse d'une sollicitation appliquée déterministe n'est donc a priori pas très forte et nous place dans un

1. $C V_{X}=\sigma_{X} / E[X]$ : coefficient de variation de $X$, rapport de l'écart-type et de l'espérance. On rappelle que l'espérance mathématique est estimée par la moyenne empirique $\bar{X}=\frac{1}{n} \sum_{1}^{n} x_{i}, x_{i}$ les réalisations de $X$, et la variance par la variance estimée $s_{\theta}^{2}(X)=\frac{1}{n-1}\left(\sum_{1}^{n} x_{i}^{2}-n \bar{X}^{2}\right)$. 
cas non conservatif, la probabilité de ruine ainsi calculée se trouvant plus faible.

Alors :

$$
\mathrm{g}=\mathrm{q}_{\mathrm{L}}-\mathrm{q}
$$

$\mathrm{q}$ déterministe, soit $\mathrm{E}[\mathrm{q}]=\mathrm{q}$ et $\sigma_{\mathrm{q}}=0$

$\beta=\frac{E\left[q_{L}\right]-q}{\sigma_{\text {qL. }}}$

Soit, avec classiquement $F=\frac{E\left[q_{L}\right]}{q}$ le coefficient de sécurité,

$$
\beta=\frac{1-1 / F}{C V_{\mathrm{qL} .}}
$$

Si on prend les valeurs couramment admises $F=3$ et $\mathrm{CV}_{\mathrm{qL} .}=40 \%$, on trouve $: \beta=1,67 \mathrm{p}_{\mathrm{t}}=4,75 \%$.

Une semelle sur vingt subirait donc des désordres dus à des défauts de portance.

Cette probabilité de ruine, beaucoup trop forte par rapport à celle observée, et qui est obtenue avec des hypothèses non conservatrices (q déterministe, pas d'accidents locaux dans le sol de fondation) a conduit quelques auteurs (ATHANASIOU-GRIVAS et HARR, 1977; BOISSIER, 1982; MC ANALLY, 1983; MOIGN, 1983) à améliorer la connaissance de la variance de $q_{t}$ en calculant celle-ci à partir de lois de distributions normales, log-normales, Beta pour les paramètres du sol et en procédant soit analytiquement par développements en série de Taylor au deuxième et quatrième ordre, soit par simulation de l'équation (7), sans pour autant obtenir une réduction sensible du coefficient de variation de $\mathrm{q}_{\mathrm{L}}$.

\section{PRISE EN COMPTE DE LA VARIABILITÉ SPATIALE}

De fait, comme la rupture des fondations superficielles intéresse de grands volumes de sol, la dispersion ponctuelle des paramètres se trouve «lissée » à l'échelle du volume concerné. FAVRE, dès 1972, posait le problème de la probabilité de rupture en terme de probabilité pour que n volumes élémentaires entrent en plasticité sous la fondation.

La variabilité spatiale, depuis les premiers travaux de VANMARCKE et FULEIHAN (1975), a été prise en compte par de nombreux auteurs pour des problèmes de tassements et stabilité à l'aide de champs stochastiques et en utilisant soit la méthode des éléments finis avec linéarisation, soit la simulation, soit les méthodes classiques de calcul lorsqu'elles n'occultaient pas l'espace. En France, il faut citer les travaux de CAMBOU (1977), de AUBRY et FROU (1979), de BAGHERY et MAGNAN (1983), de MADHAVI (1985) et de BOULEFKHAD (1986).

Seul GENEVOIS (1984) a traité le problème des fondations superficielles, qui présente des difficultés particulières :

- on ne peut définir une surface matérialisée de cisaillement;

- le passage des enfoncements d'une fondation superficielle aux états de contraintes limites est une transformation fortement non linéaire à cause des grandes déformations qui se manifestent au moment de la rupture;
- la simulation classique dans une procédure de calcul par éléments finis utilisant une loi élastoplastique pour le sol est extrêmement coûteuse, vu la nécessité de simuler tout le chargement jusqu'à la rupture.

On a donc défini une méthode simplifiée de simulation utilisant un calcul en éléments finis, seul capable, à l'heure actuelle, de prendre en compte les champs stochastiques dans les problèmes de portance des fondations superficielles. L'étude montre qu'on obtient des coefficients de variation dix à cent fois plus faibles qu'en traitant directement l'équation (7) de la capacité portante.

\section{PROBLÈME ÉTUDIÉ ET PROCÉDURES}

L'étude porte sur une semelle filante, non encastrée, reposant sur un massif de sable sec.

On a utilisé le code de calcul par éléments finis « Aubry. Des Croix-Hujeux * du laboratoire de l'École Centrale de Paris, avec une loi de Drucker-Prager à potentiel associé pour prendre en compte la dilatance.

DES CROIX (1980) a montré que l'on retrouvait le coude de la courbe scharge-enfoncement "entre la charge limite donnée par le calcul de Terzaghi et celle donnée par le calcul de Prandtl-Caquot dès que les rapports de la hauteur $\mathrm{H}$ et de la longueur $\mathrm{L}$ du maillage à la largeur $\mathrm{B}$ de la fondation étaient respectivement de six et onze.

La nécessité d'avoir un nombre suffisant d'éléments pour prendre en compte l'hétérogénéité du sol et des dimensions de maillage suffisantes pour retrouver le coude de la courbe a charge-enfoncement " entrait en contradiction avec la nécessité d'avoir des temps de passage courts, ces passages étant en grand nombre. On a donc adopté la procédure suivante :

a. le chargement se fait en enfoncements imposés suivant un pas régulier, la charge étant définie par intégration des composantes verticales de la contrainte sous la fondation;

b. un carré de quatre-vingt-un éléments égaux de côté $\mathrm{B} / 3$, appelé noyau, est placé sous la fondation. Les dimensions du maillage sont agrandies $(\mathrm{H} / \mathrm{B}=15$, $\mathrm{L} / \mathrm{B}=24$ ) mais le noyau est complété avec de grands éléments. On obtient ainsi cent-soixante-neuf éléments (fig. 1) ;

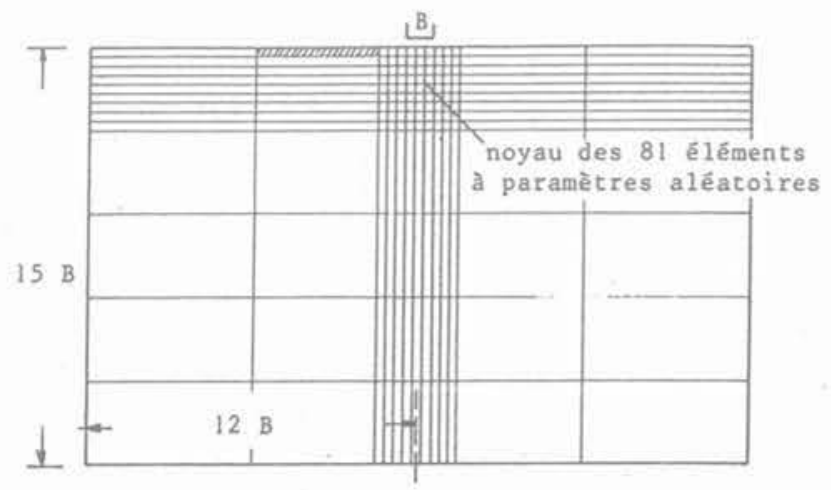

Fig. 1. - Maillage du problème. 
c. la charge limite est celle à partir de laquelle les incréments de charge changent de domaine de variation pour devenir faibles.

De nombreux tests ont été exécutés pour trouver un compromis entre le coût du passage et une bonne caractérisation du coude de la courbe «charge-enfoncement».

\section{ESTIMATION DES PARAMÈTRES DE CHARGE LIMITE ET SIMULATION DE LA VARIABILITÉ SPATIALE}

Pour éviter des temps de calcul trop longs lors de la simulation de la variabilité spatiale des paramètres, la procédure suivante en trois étapes a été utilisée (fig. 2) : - avec les valeurs moyennes des paramètres de sol dans tout le maillage (étape 1), on détermine l'intervalle des enfoncements correspondant à la charge limite, à l'aide d'un pas moyen d'enfoncement (pour $\varnothing=30^{\circ} \Delta e=1 \mathrm{~cm}$ ), puis :

- (étape 2) on détermine l'enfoncement correspondant à la charge critique, avec un grand pas $\Delta e=2,5 \mathrm{~cm}$, jusqu'à la borne inférieure de l'intervalle précédent puis, à l'aide d'un petit pas $(\Delta e=0,1 \mathrm{~cm})$. Cette étape, faite avec beaucoup d'itérations pour éviter les effets de décompression du maillage (fig. 3), est très coûteuse ;

- avec les valeurs simulées des paramètres de sol affectées aux éléments du maillage, (étape 3) on va jusqu'à l'enfoncement limite en deux ou trois pas avec peu d'itérations. On détermine la charge correspondante, qui sera une réalisation de la charge limite.

On répète l'étape 3 autant de fois qu'on veut de réalisations de la charge limite.

Cette procédure objective ne nécessite qu'un passage coûteux par cas étudié. Elle introduit un léger biais sur l'estimation de la moyenne de la charge limite $q_{L}$, mais le but poursuivi est de montrer que sa variance est bien plus petite que celle trouvée par des calculs classiques. Toujours dans le but de diminuer les coût de calcul, nous avons testé la sensibilité de la charge limite à de faibles valeurs des paramètres du sol dans des éléments les plus proches de la semelle. On obtient les résultats suivants présentés dans le tableau 1.

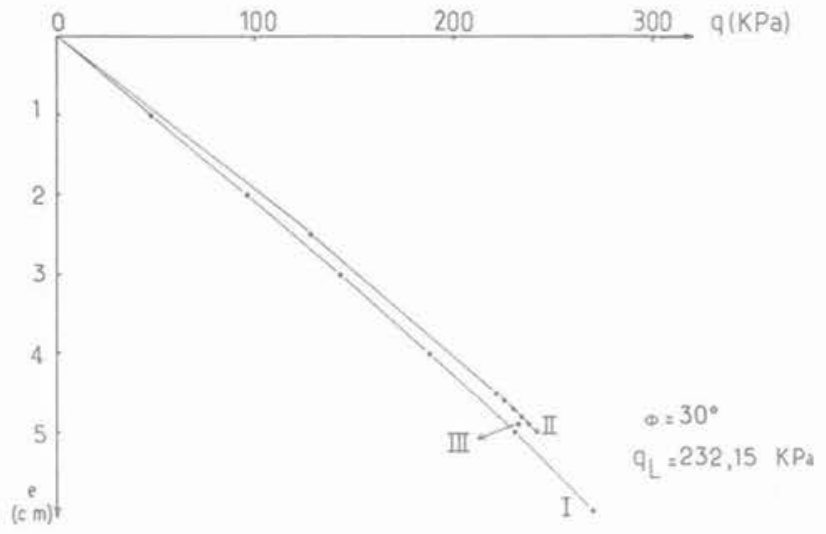

Fig. 2. - Etapes du chargement.

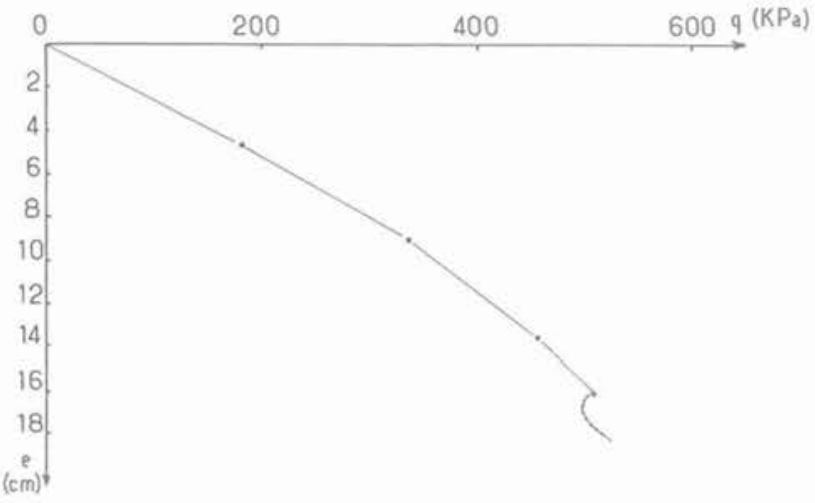

Fig. 3. - Effet de décompression du maillage.

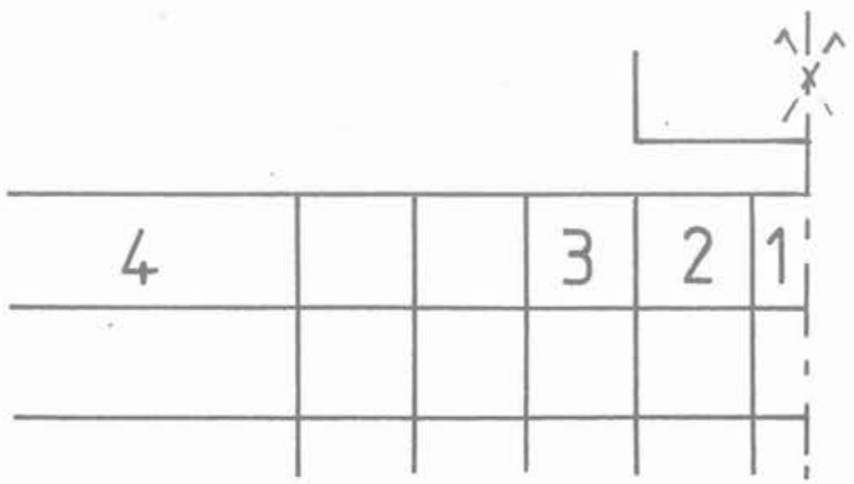

avec $\varnothing=30^{\circ}, \mathrm{E}=20 \mathrm{MPA}, \mathrm{CV}_{\varnothing}=20 \%: \quad \overline{\mathrm{q}}_{\mathrm{L}}=231,21 \mathrm{KPa}, \mathrm{s}_{\mathrm{qL}}^{\mathrm{e}}=1,899 \mathrm{KPa}$. 


\section{CAS ÉTUDIÉS ET CARACTÉRISATION DES VARIABLES SIMULÉES}

L'étude s'est faite avec $B=1,5 \mathrm{~m}$; les éléments du noyau sont des carrés de $50 \mathrm{~cm}$ de côté.

Les caractéristiques prises par un charnp de moyenne locale sur le pas d'espace $50 \mathrm{~cm} \times 50 \mathrm{~cm}$ sont proches de celles du champ «ponctuel , le «point » ayant la taille des échantillons $10 \mathrm{~cm} \times 20 \mathrm{~cm}$ sur lesquels sont mesurés classiquement les paramètres mécaniques des sables. Ainsi, on a adopté pour les deux paramètres aléatoires du modèle $\mathrm{E}$ : module d'élasticité, $\varnothing$ : angle de frottement interne de plasticité, des caractéristiques conformes à la littérature internationale. On se place donc dans un cas conservatif ne tenant pas compte de la fonction de variance qui mesure la réduction de la variance ponctuelle (définie ici sur un espace d'environ $10 \mathrm{~cm} \times 20 \mathrm{~cm}$ ) par moyenne locale (ici sur un espace $50 \mathrm{~cm} \times 50 \mathrm{~cm}$ ).

Plusieurs auteurs ont proposé des plages de valeurs moyennes et de coefficients de variation pour l'angle de frottement et le module d'élasticité des sables. FAVRE (1980) et MAGNAN (1982) en ont dressé un répertoire assez complet.

Par contre, il existe très peu d'études sur la corrélation entre $E$ et $\varnothing$ et sur leur autocorrélation (ALONSO et KRIZEK, 1975; LUMB, 1975; VANMARCKE, 1977). Nous avons adopté, pour le coefficient d'autocorrélation, le modèle exponentiel simple :

$$
\begin{array}{r}
\rho[E(x), E(x+\Delta x)] \\
=\frac{E[\{(x+\Delta x)-\bar{E}(x+\Delta x)\{\{E(x)-\bar{E}(x)\}]}{\operatorname{VAR}^{1 / 2}[E(x+\Delta x)] \operatorname{VAR}^{1 / 2}[E(x)]} \\
=\frac{\operatorname{COV}[E(x), E(x+\Delta x)]}{\sigma E(x) \sigma E(x+\Delta x)}
\end{array}
$$

(on note abusivement $\bar{E}(\mathrm{x})=\mathrm{E}[\mathrm{E}(\mathrm{x})]$ pour alléger l'écriture, avec $E(x)$ le module d'élasticité dépendant de la coordonnée d'espace $\mathrm{x}$ ).

$=p(\Delta x)=e^{-\alpha}|\Delta x|$ (champ homogène indépendant dex).

pour lequel $\theta$, échelle de fluctuation, est égale à $2 / \alpha$, et procédé à une étude de sensibilité en examinant vingt-cinq cas couvrant les plages suivantes :

$$
\begin{gathered}
\varnothing \text { de } 30 \text { à } 40^{\circ}, C V_{\odot} \text { de } 10 \text { à } 20 \%, \\
E=20 \mathrm{MPa}, \mathrm{CV}_{\mathrm{E}} \text { de } 0 \text { à } 30 \%
\end{gathered}
$$

$\varnothing$ et $\mathrm{E}$ sont gaussiens.

Premier groupe de cas : $\varnothing$ aléatoire, E déterministe, $\varnothing$ non corrélé et corrélé verticalement et horizontalement d'un élément à l'autre avec, comme échelles de fluctuation :

$\theta_{\mathrm{h}}=1,2$ et $7 \mathrm{~m}$ et $\theta_{v}=1-1,6$ et $2 \mathrm{~m}$

Deuxième groupe de cas : $\varnothing$ aléaoire, E aléatoire, pas d'autocorrélation d'un élément à l'autre $\theta=1 \mathrm{~m}$ corrélation entre $\varnothing$ et $E: \rho=0-0,3$ et 0,7 .

- On a contrôlé, par analyse statistique multivariable, que les huit tirages des quatre-vingt-une valeurs aléatoires du noyau étaient bien indépendants : généralement $70 \%$ des coefficients de corrélations entre tirages étaient inférieurs à $10 \%$ et les autres étaient inférieurs à $20 \%$.
- Les valeurs des paramètres tirées dans les lois de distribution continues ont été ramenées à sept valeurs types: $x, x(1 \pm C V / 2), x(1 \pm C V), x(1 \pm 3 C V / 2)$, par classes de largeur $\times . C V / 2$.

Les valeurs $|X|>1,75 \sigma$ ont été ramenées à $\pm 1,5 \sigma$ : on a environ trois éléments dans ce cas par tirage et l'on a vu que leur poids était faible sur la variation de $\mathrm{q}_{1}$ hormis pour un seul élément (tabl. I).

On a donc jugé que cette simplification n'avait pas de rôle significatif sur les réalisations de la charge limite.

\section{RÉSULTATS}

Le tableau II regroupe des valeurs de l'écart-type de la charge ultime pour les vingt-cinq cas étudiés.

Les figures 4,5 et 6 indiquent les variations du coefficient de variation de la charge ultime en fonction des différents paramètres statistiques.

On constate que :

- la dispersion des charges $C V_{\text {oL }}$ est peu sensible aux variations de la moyenne de $\varnothing$ (fig. 4);

- l'autocorrélation ne joue pas un rôle aussi sensible qu'on aurait pu le penser; pour $\alpha=0,3$, qui correspond à une corrélation de 0,63 entre les extrémités de la semelle, on ne fait que doubler la dispersion des charges (fig. 5);

- l'introduction d'une deuxième variable joue un rôle prépondérant: $\mathrm{CV}_{\mathrm{qL}}$ est multiplié par huit quand on passe d'une dispersion nulle à une dispersion de $30 \%$ de $E$ et la corrélation joue un rôle faible (fig. 6).

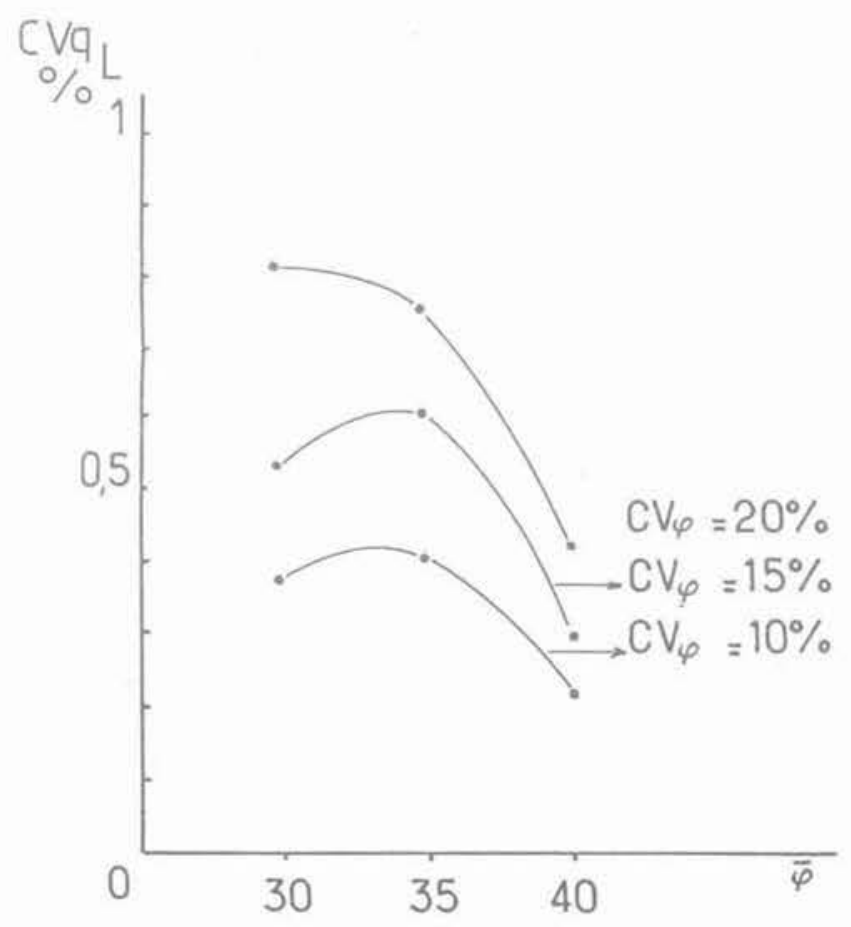

Fig. 4. - Sensibilité de $\mathrm{CV}_{q}$ à un seul paramètre aléatoire : l'angle de frottement interne $\varnothing$. 


\begin{tabular}{|c|c|c|c|c|c|c|c|c|c|c|c|c|c|}
\hline & \multicolumn{3}{|c|}{$\bar{q}=235 \mathrm{KPa}$} & \multicolumn{3}{|c|}{$\overline{\mathrm{q}}=527 \mathrm{KPa}$} & \multicolumn{3}{|c|}{$\bar{q}=1275 \mathrm{KPa}$} & & & \\
\hline & & \multicolumn{3}{|c|}{$\phi=30^{\circ}$} & \multicolumn{3}{|c|}{$\varphi=35^{\circ}$} & \multicolumn{3}{|c|}{$\theta=40^{\circ}$} & & & \\
\hline & & $\begin{array}{l}\mathrm{CV}= \\
10 \%\end{array}$ & $\begin{array}{l}\mathrm{CV}= \\
15 \%\end{array}$ & $\begin{array}{l}\mathrm{CV}= \\
20 \%\end{array}$ & $\begin{array}{l}\mathrm{CV}= \\
10 \%\end{array}$ & $\begin{array}{l}\mathrm{CV}= \\
15 \%\end{array}$ & $\begin{array}{l}\mathrm{CV}= \\
20 \%\end{array}$ & $\begin{array}{l}\mathrm{CV}= \\
10 \%\end{array}$ & $\begin{array}{l}\mathrm{CV}= \\
15 \%\end{array}$ & $\begin{array}{l}C V= \\
20 \%\end{array}$ & \multicolumn{2}{|c|}{$\begin{array}{l}\text { autocorrélation } \\
\text { de p }\end{array}$} & $\begin{array}{l}\text { corrélation } \\
\text { entre } \varphi \text { et } E\end{array}$ \\
\hline \multirow{11}{*}{$\begin{array}{l}\mathrm{E}= \\
20 \mathrm{MPa}\end{array}$} & \multirow{5}{*}{$\begin{array}{l}\mathrm{CV}_{\mathrm{E}}= \\
0\end{array}$} & 0,887 & 1,247 & 1,899 & 2,176 & 3,205 & 3,974 & 2,809 & 3,715 & 5,314 & $\frac{1}{a h}=0$ & nulle & \multirow{5}{*}{ E constant } \\
\hline & & 1,428 & & 2,183 & & & & 3,767 & & 7,942 & $\frac{1}{a h}=1,11$ & \multirow{2}{*}{$\begin{array}{l}\text { mono- } \\
\text { dimens: }\end{array}$} & \\
\hline & & 2.459 & & 3,401 & & & & 5.272 & & 10,315 & $\frac{1}{a h}=3,33$ & & \\
\hline & & 2,306 & & & & & & & & & $\begin{array}{l}1 / a h=3,33 \\
1 / a^{v}=1,11\end{array}$ & \multirow{2}{*}{ dimens. } & \\
\hline & & 2,079 & & & & & & & & & $\begin{array}{l}1 / a n=1,11 \\
1 / a^{y}=0,83\end{array}$ & & \\
\hline & \multirow{3}{*}{$\begin{array}{l}\mathrm{CV}_{\mathrm{e}}= \\
10 \%\end{array}$} & 3.287 & & & & & & & & & \multirow{6}{*}{$\frac{1}{a \mathrm{~h}}=0$} & \multirow{6}{*}{ nulle } & $p=0$ \\
\hline & & 3,309 & & & & & & & & & & & $p=0,3$ \\
\hline & & 3.444 & & & & & & & & & & & $p=0,7$ \\
\hline & \multirow{3}{*}{$\begin{array}{l}\mathrm{CV}_{\mathrm{E}}= \\
30 \%\end{array}$} & 7,470 & & & & & & & & & & & $\rho=0$ \\
\hline & & 8,317 & & & & & & & & & & & $p=0,3$ \\
\hline & & 12,530 & & & & & & & & & & & $\rho=0,7$ \\
\hline
\end{tabular}

Tableau II. - Valeurs des écarts-types de $q_{L}$ pour les vingt-cinq cas étudiés (en KPa).

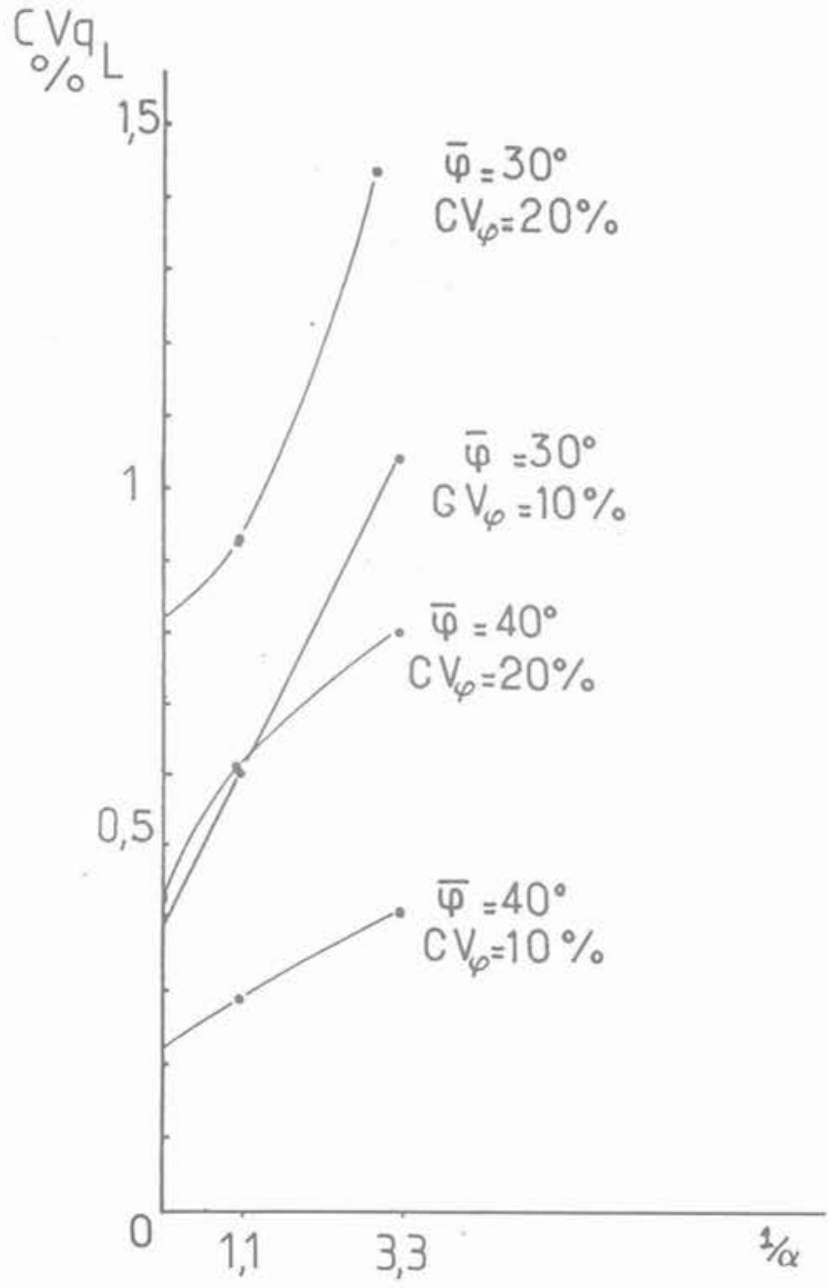

Fig. 5. - Sensibilité de $C V_{q_{L}}$ à l'échelle de fluctuation horizontale $\theta_{h}$ de l'angle de frottement.

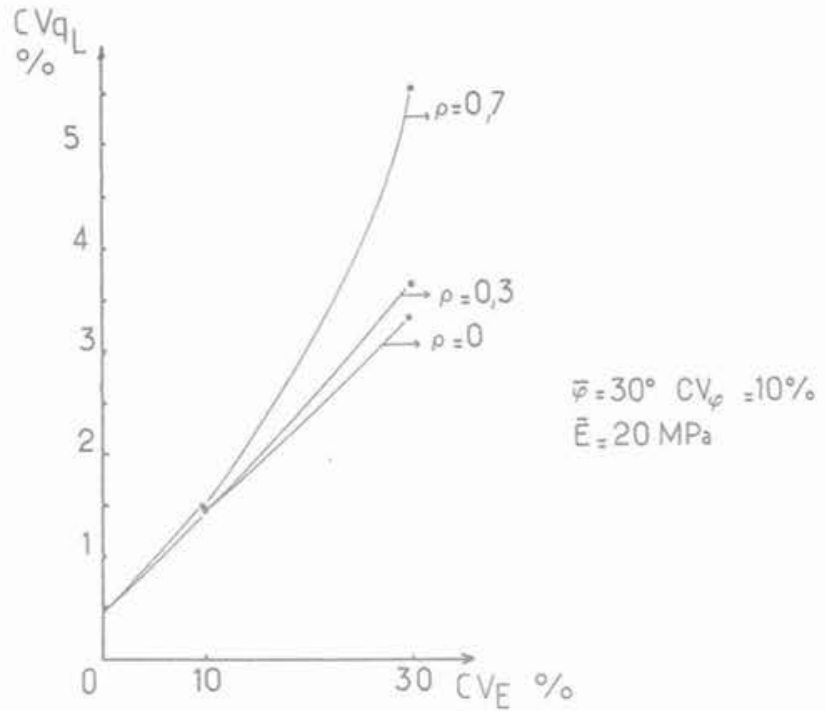

Fig. 6. - Sensibilité de $C V_{q L}$ à deux paramètres aléatoires corrélés : I'angle de frottement interne $\varnothing$ et le module d'élasticité $E$.

On retiendra que $\mathrm{CV}_{\mathrm{qL}}$ est d'abord sensible au nombre de variables aléatoires du sol et que c'est la dispersion de ces variables de base qui joue un rôle et non leur valeur moyenne.

L'extrapolation de nos résultats, dans nos plages de valeurs et sans autocorrélation sur $\mathrm{E}$, conduirait au cas le plus défavorable suivant :

$\mathrm{CV}_{\text {qL }}<10 \% \varnothing$ de 30 à $35^{\circ}, \mathrm{CV}_{\varnothing}=20 \%$,

$$
\begin{aligned}
& \theta_{\varnothing, \mathrm{h}}=7 \mathrm{~m}, \theta_{\varnothing, v}=1,6 \mathrm{~m} \\
& \mathrm{E}=20 \mathrm{MPa}, C V_{E}=30 \%, \\
& \theta_{\mathrm{E}, \mathrm{h}}=\theta_{\mathrm{E}, \mathrm{v}}=1 \mathrm{~m}
\end{aligned}
$$




\section{REPRÉSENTATIVITÉ DES RÉSULTATS OBTENUS}

L'estimation de l'espérance et de l'écart-type de la charge ultime à l'aide de huit valeurs seulement pose le problème majeur de la confiance que l'on peut avoir dans les résultats trouvés et dans l'indice de sécurité estimé.

Nous donnant un seuil inférieur pour l'indice de sécurité (la probabilité de ruine augmente quand celui-ci diminue), nous avons calculé une borne supérieure à la probabilité que $\beta$ a de lui être inférieur.

La normalité de $q_{L}$ a été vérifiée par test de ShapiroWilk.

En notant désormais $s_{e}=s_{e}\left(q_{1}\right)$, l'écart-type estimé, pour alléger l'écriture,

- soit $A$, l'événement * $E\left[q_{1}\right] \leqslant q \min$,

avec $\mathrm{q} \min =\overline{\mathrm{q}}_{\mathrm{L}}-\mathrm{t}_{a, \mathrm{n}-1} \mathrm{~s}_{\mathrm{e}} / \sqrt{\mathrm{n}}$

$\mathrm{t}_{\alpha, n-1}$ : valeur que la variable de Student, à $n-1$ degrés de liberté, a $\alpha$ chance de dépasser,

- soit $B$, l'événement $\alpha \sigma_{\mathrm{qL}}>s_{e} \max$,

avec $s_{e} \max =s_{e}\left[(n-1) / \chi_{1-a, n-1}^{2}\right]^{1 / 2}$

$\chi_{1-a, n-1}^{2}$ : valeur que la variable du $\chi^{2}$, à $n-1$ degrés de liberté, a $1-\alpha$ chance de dépasser,

la théorie de l'estimation nous permet d'écrire :

$$
\mathrm{P}(\mathrm{A})=\alpha ; \mathrm{P}(\mathrm{B})=\alpha .
$$

L'équation (10) nous donne, avec $F=3$ :

$$
\beta=\frac{2}{3} E\left[q_{L}\right] / \sigma_{\mathrm{qL}} \text {. }
$$

Soit le seuil $\beta \min =\frac{2}{3} q_{L} \min / \mathrm{s}_{e} \max$.

La probabilité d'avoir $\beta<\beta$ min peut être représentée par la probabilité que le point de coordonnées ( $E\left[q_{L}\right]$, $\sigma_{\text {qL }}$ ) soit dans le domaine $\bar{D}$ (graphique a).

La probabilité d'avoir les événements A ou $\mathrm{B}$ (on note $A \cup B$ ) peut être représentée par le domaine $\bar{\Delta}$ (graphique b).

D'où, $\operatorname{Prob}\{\beta<\beta \min \}<P(A \cup B)$

D'après la théorie des probabilités, on peut écrire :

$P(A \cup B)=P(A)+P(B)-P(A \cap B)$.

$A \cap B$ est l'événement «avoir $A$ et $B$ *.

$$
\begin{gathered}
\mathrm{P}(\mathrm{A})=\mathrm{P}(\mathrm{B})=\alpha, \mathrm{P}(\mathrm{A} \cap \mathrm{B}) \geqslant 0, \\
\text { donc } \mathrm{P}(\mathrm{A} \cup \mathrm{B}) \leqslant 2 \alpha, \\
\text { Prob }\{\beta<\beta \min \}<2 \alpha
\end{gathered}
$$

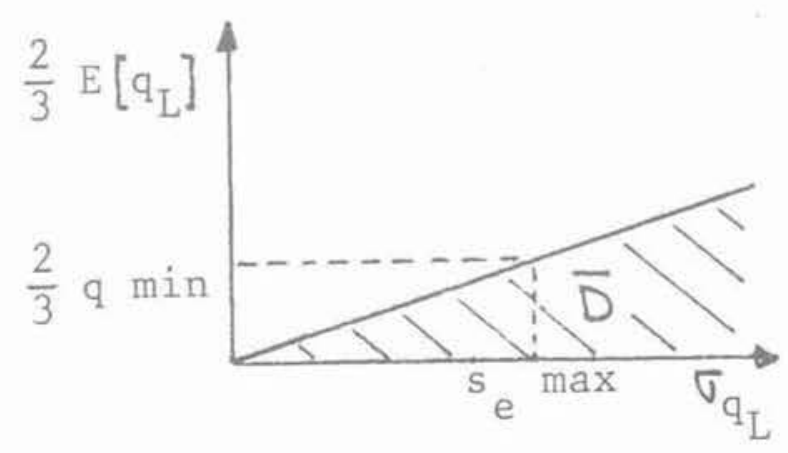

graphique a

$$
\begin{aligned}
& \text { Soit, avec } \alpha=0,10 \mathrm{n}=8 \mathrm{t}_{10 \%, 7}=1,415 \\
& \chi_{90 \%, 7}^{2}=2,833 \text {, } \\
& \beta \min =\left(1 / \mathrm{CV}_{\mathrm{QL} .}-0,500\right) 0,424 \\
& \text { pour } \mathrm{CV}_{\mathrm{qL}}=0,22 \%\left(\bar{\varnothing}=40^{\circ}, \mathrm{CV}_{\varnothing}=10 \%\right. \text {, } \\
& \theta_{\varnothing, \mathrm{h}}=\theta_{\varnothing, \mathrm{v}}=1 \mathrm{~m}, \mathrm{E}=20 \mathrm{MPa} \\
& \text { Prob }\{\beta \leqslant 192\}<0,20 \\
& \text { pour } \mathrm{CV}_{\text {q. }}=2,30 \%\left(\bar{\varnothing}=30^{\circ}, \mathrm{CV}_{\varnothing}=10 \%\right. \text {, } \\
& \left.\theta_{\emptyset, \mathrm{h}}=7 \mathrm{~m}, \theta_{\emptyset, \mathrm{v}}=2 \mathrm{~m}, \mathrm{E}=20 \mathrm{MPa}\right) \\
& \text { Prob }\{\beta \leqslant 18,2\}<0,20 \\
& \text { pour } \mathrm{CV}_{\mathrm{qL}}=5,53 \% \\
& \begin{array}{c}
\overline{\bar{D}}=30^{\circ}, \mathrm{CV} \oslash=10 \%, \theta_{\varnothing, \mathrm{y}}=1 \mathrm{~m} \\
\overline{\mathrm{E}}=20 \mathrm{MPa}, \mathrm{CV}_{\mathrm{E}}=30 \%, \theta_{\mathrm{E}, \mathrm{h}}=\theta_{\mathrm{E}, \mathrm{v}}=1 \mathrm{~m} \\
\text { Prob }\{\beta \leqslant 7,46\}<0,20
\end{array}
\end{aligned}
$$

Pour $\mathrm{CV}_{\text {qL. }} 10 \%$, notre cas extrême extrapolé, on obtient :

$$
\begin{gathered}
\bar{\varnothing} \text { de } 30 \text { à } 35^{\circ}, C V_{\varnothing}=20 \%, \theta_{\varnothing, \mathrm{h}}=7 \mathrm{~m}, \theta_{\varnothing, \mathrm{v}}=1,6 \mathrm{~m} \\
\overline{\mathrm{E}}=20 \mathrm{MPa}, \mathrm{CV}_{\mathrm{E}}=30 \%, \theta_{\mathrm{E}, \mathrm{h}}=\theta_{\mathrm{E}, \mathrm{v}}=1 \mathrm{~m} \\
\operatorname{Prob}\{\beta \leqslant 4,03\}<0,20 \\
\Phi(-4,03)=310^{-5}
\end{gathered}
$$

On a donc deux chances sur dix d'obtenir une probabilité de ruine inférieure à $310^{-5}$.

\section{CONCLUSIONS}

Lorsque la comparaison est possible, nous trouvons des dispersions de dix à cent fois plus petites que celles trouvées par d'autres auteurs qui utilisent l'équation de la capacité portante classique.

La dispersion des paramètres de sol n'a d'effet important sur la variabilité de la force portante des fondations superficielles que dans des cas extrêmes : fortes dispersions des paramètres, fortes corrélations entre eux, échelles de fluctuation importantes par rapport à la dimension de la fondation superficielle.

Les analyses classiques sont impropres à donner une idée des probabilités de ruine des fondations superficielles. Seules les procédures prenant en compte la variabilité spatiale peuvent approcher la réalité.

Notre estimation est tout à fait grossière puisque les charges sont considérées déterministes ainsi que les autres paramètres du sol et autres facteurs d'incertitudes comme les dimensions de la semelle, l'hétérogé-

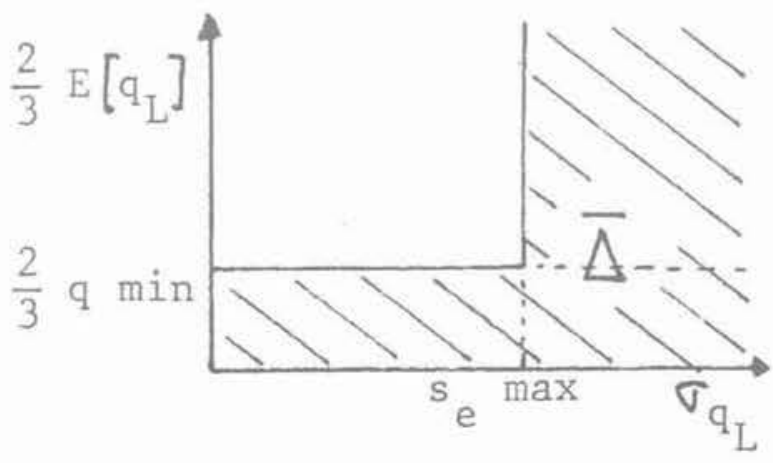

graphique b 
néité locale, la variabilité dans le temps, le rôle de l'eau, etc.

Notre but n'était pas de calculer la probabilité de ruine, mais de montrer que la prise en compte de la variabilité spatiale des paramètres du sol induit des dispersions plus faibles de la capacité portante que celles trouvées par des calculs directs.

Cette moindre dispersion induit des probabilités de ruine beaucoup plus petites et nous ramènerait dans le domaine des phénomènes observés, en prenant en compte toutes les autres incertitudes.

Le coût ordinateur de la mise au point des procédures et des vingt-cinq cas étudiés a été environ de 50000 francs.

\section{BIBLIOGRAPHIE}

1. ALONSO E., KRIZEK R. (1975), Stochastic formulation of soil properties. Proc. $2^{\text {nd }}$ I.C.A.S.P. vol. II, pp. 9-33, Aachen.

2. ATHANASIOU-GRIVAS D., HARR M. (1977), Reliability with respect to bearing capacity failures of structures on ground. 9e C.I.M.S.T.F., Tokyo 1977, C.R. 6e session spéciale, 25 p. éd. École Centrale de Paris.

3. AUBRY D. et FROU P. (1983), Finite element analysis of the stochastic elastodynamic equation with special reference to earthquake loading. Proc. $3^{\text {rd }}$ I.C.A.S.P., vol. II, pp. $748-755$. Sydney.

4. BAGHERY S., MAGNAN J.P. (1983), Analyse probabiliste de la stabilité et des tassements des remblais du site expérimental de Cubzac-Les. Ponts. L.C.P.C. Paris. Rapport de Recherches L.P.C., n० 122,69 p.

5. BENJAMIN J.R., CORNELL C.A. (1970), Probability, Statistics and Decision for Civil Engineers. Mc Graw-Hill, New York.

6. BOISSIER D. (1982), Contribution à la prise en compte des interactions Sols-Fondations-Bâti dans la conception des bâtiments. Approche Probabiliste de la Sécurité». Thèse D. Es. Sces. I.N.S.A., Lyon.

7. BOULEFKHAD T. (1986), Influence de la variabilité des propriétés des sols sur le calcul des tasse. ments des fondations superficielles. Thèse D. Ing. Univ. Clermont II.

8. CAMBOU B. (1977), L'incertitude sur les résultats d'un problème de mécanique des sols ou des roches traité par la méthode des éléments finis. Revue Française de Géotechnique, juillet 1977. $\mathrm{n}^{\circ} 1, \mathrm{pp} .55-64$.
9. DES CROIX Ph. (1980), Intégration numérique d'une loi de comportement élastoplastique à deux seuils. Thèse D. Ing. École Centrale de Paris.

10. FAVRE J.L. (1972), Pour un traitement par le calcul de probabilité et statistiques des problèmes de mécanique des sols. Thèse D. $3^{e}$ cycle, univ. de Grenoble.

11. FAVRE J.L. (1980), Milieu continu et milieu discontinu : Mesure statistique indirecte des paramètres rhéologiques et approche probabiliste de la sécurité. Thèse D. Es Sces., univ. P. et M. Curie, Paris.

12. GENEVOIS B. (1984), Rôle de la variabilité spatiale des paramètres de sol sur la capacité portante des fondations superficielles. Thèse D. Ing. Ecole Centrale de Paris.

13. HASOFER A et LIND N. (1974), An exact and invariant first order reliability format, Journal of Eng. Mech. Div. A.S.C.E., New York, 100, E.M.I., pp. 111-121.

14. LUMB P. (1975), Spatial variability of soils properties. Proc. $2^{\text {nd }}$ I.C.A.S.P., vol. II, pp. 397-422, Aachen.

15. MC ANALLY P. (1983), Reliability of the bearing capacity desing of shallow footing in the sands. Proc. $4^{\text {th }}$ I.C.A.S.P., vol. II, pp. 1545-1557, Firenze.

16. MAHDAVI C. (1985), Analyse probabiliste du comportement des sols et des ouvrages. Évaluation des risques dans les études géotechniques de tracés de remblais sur sols mous. Thèse D. Ing. E.N.P.C., Paris.

17. MAGNAN J.P. (1982), Les méthodes statistiques et probabilistes en mécanique des sols. Presses E.N.P.C., Paris.

18. MAGNAN J.P. et BAGHERY S. (1982). Statistiques et probabilités en mécanique des sols - État des connaissances. L.C.P.C. Paris. Rapport de Recherches L.P.C. $n^{\circ} 109,191$ p.

19. MEYERHOF G.G. (1977), Partial and total safety factors. Rapport des organisateurs. Session spéciale $n^{\circ} 6$ : L'approche probabiliste dans les études de Mécanique des Sols. 9e C.I.M.S.T.F., Tokyo, 1977, vol. 3. pp. 503-505.

20. MOIGN H. (1983), La méthode des moments pour le calcul de la force portante des fondations. Rapport de D.E.A., École Centrale de Paris.

21. SCHULTZE E. (1977), L'approche probabiliste dans les études de Mécanique des Sols. Rapport des organisateurs. Session spéciale $n^{\circ} 6$, 9e C.I.M.S.T.F., Tokyo, vol. 3, pp. 501-503.

22. VANMARCKE E. et FULEIHAN N. (1975), Probabilistic prediction of levee settlements. Proc. $2^{\text {nd }}$ I.C.A.S.P., vol. II, pp. 175-190, Aachen.

23. VANMARCKE E. (1977), Probabilistic Modeling of Soils Profiles. Journal of Geotech. Eng. Div. A.S.C.E. GT II, pp. 1227-1246. 